\title{
STUDY OF METHODOLOGY FOR QUANTITATIVE THERMAL DIAGNOSTIC OF WALL
}

\author{
Y.YANG $^{127^{+*}}$, A. SEMPEY ${ }^{123}$, T. Vogt-WU ${ }^{123}$, A. SOMMIER ${ }^{213}$, J. DUMOULIN $^{45}$, C. \\ PRADERE $^{213}$, J.C. BATSALE ${ }^{321}$ \\ ${ }^{l}$ Univ. Bordeaux, I2M, UMR 5295, F-33400 Talence, France. \\ ${ }^{2}$ CNRS, I2M, UMR 5295, F-33400 Talence, France. \\ ${ }^{3}$ Arts et MetiersParisTech, I2M, UMR 5295, F-33400 Talence, France. \\ ${ }^{4}$ IFSTTAR, COSYS-SII, F-44344, Bouguenais, France \\ ${ }^{5}$ Inria, I4S Team, Campus de Beaulieu, F-35042 Rennes, France \\ +*Presenting and Corresponding Author: yingying.yang@u-bordeaux.fr
}

\begin{abstract}
Non-destructive evaluation (NDE) method has been popularly used for thermal diagnosis of building envelop in order to retrofit old buildings, detect civil engineering structures and accredit new buildings. The infrared thermography technique has been widely applied in the NDE method. In order to develop new solutions for diagnostic of local thermal performance, experiments were carried out on two cases of multilayer walls. Experimental results of emissivity, temperature, and heat fluxes will be shown and analyzed in this article. The main originality of this work is to try to compute the front face temperature response to transient periodic heating by computing the front face pulse response. Such front face pulse response is obtained by a deconvolution method and a TSVD inverse method. The thermal properties of the wall will be characterized through the opotimization method based on the thermal quadrupoles model.
\end{abstract}

KEYWORDS: IR thermography, thermal properties, wall, non-destructive evaluation (NDE), truncated singular value decomposition (TSVD), thermal quadrupoles model

\section{INTRODUCTION}

Energy consumption of building takes the largest share in the energy structure (35\% of total final energy consumption), and it is also responsible for approximately one-third of global carbon emissions[1]. The building envelope is the critical role in determining the building efficiency and the levels of comfort. The thermal diagnostic of the building envelope is of great importance in the cases of new building accreditation, energy efficiency renovation of existing old building, and building resale. However, there are some difficulties for applying the conventional thermal diagnostic methodologies [2] (for example, the flash method) on the building in situ because of the large thickness of the wall and the uncontrolled conditions. Nowadays, the infrared thermography technology has been widely employed in the nondestructive evaluation (NDE) of building for both qualitative and quantitative diagnostic[3], [4]. The analysis of numerical modelling based on transient heat transfer regime becomes a hot topic.

In this study, the infrared thermography is applied for emissivity measurement and temperature measurement. The front face pulse response is computed by the TSVD. The thermal quadrupoles model[5] and inverse method are applied to characterize the thermal properties of the wall.

\section{EXPERIMENT}

The experiments were carried out in two cases. Case 1 is conducted in the laboratory, a multi-layer practical scale wall that fixed on a caisson placed in a climatic chamber was detected. The insulation layer of the wall is behind the concrete block layer. Case 2 is conducted on a building in situ, an exterior wall of a building in Bordeaux was diagnostic, the insulation layer of the wall is in front of the concrete block layer. In the two cases, the objective wall has been instrumented with heat flux and temperature sensors and 
heated by lamps. The IR camera (FLIR A325) is fixed in front of the walls to take infrared images during the test.

\section{RESULTS AND ANALYSIS}

First, the emissivity of the wall surface and treated sensor surfaces were evaluated by using an IR camera with an adapted post-processing based on the standard ISO 18434, as shown in Figure 1. The measured emissivity of wall matches well with the reference values.

Next, Comparison is made between the temperature that measured by the IR camera and temperature that measured by thermocouples. As shown in Fig.2, normally this temperature difference is acceptable in the thermal characterization evaluation of building in situ.

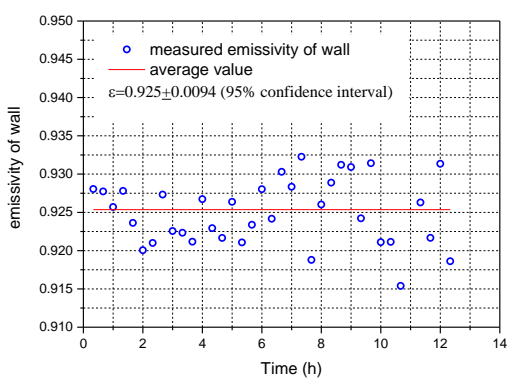

Fig.1 Measured emissivity of wall

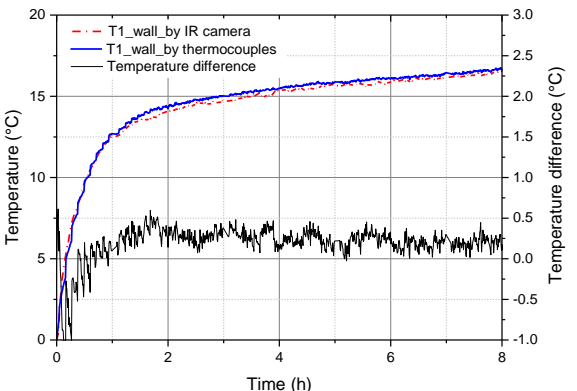

Fig.2 Comparison of temperature by IR camera and by thermocouple

Then, in the case of transient regime heat transfer measurement, the objective wall is heated by several different perodic heating signals. Both the temperature and heat flux of front face (the heated surface) are measured by sensors. The front face pulse response can be calculated by the temperature curves and heat flux of front face of the wall. Truncated singular value decomposition (TSVD) method is used in this calculation for the deconvolution. The front face pulse response has also been simulated by thermal quadrupoles model. As shown in Fig.3, the experimental values match well with the simulated value. Left figure is the results of Case 1 and right figure is the results of Case 2.
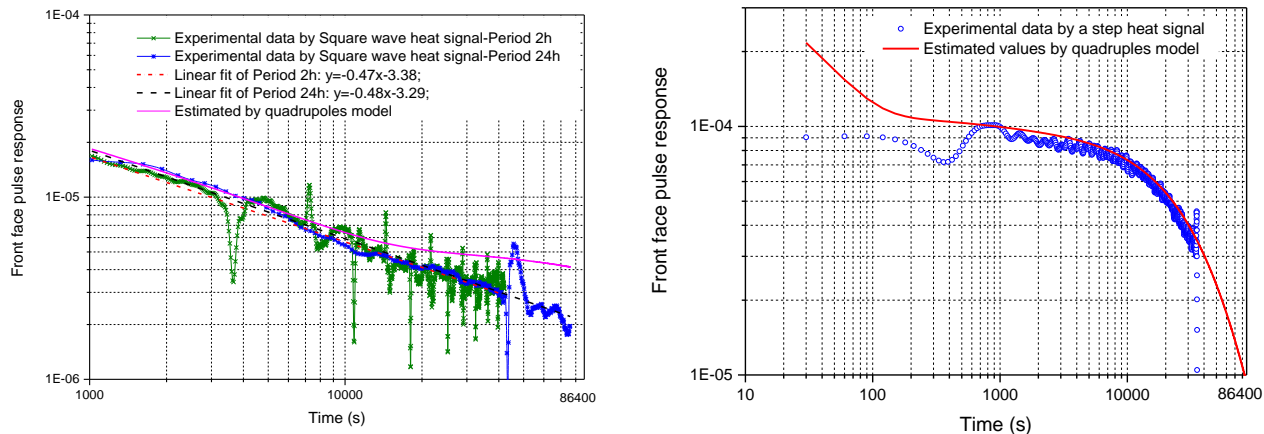

Fig.3 Front face pulse response: experimental calculated values VS simulated values by thermal quadruples model. (left: Case 1; right: Case2)

Finally, with the measured heat flux of wall, the temperature of the wall can be predicted by the thermal quadrupole method. The optimum values of thermal parameters are estimated by minimiazing the measured temperature and predicted temperature. The optimization results have a good agreement with the values from the references, the mean relative error is $13.09 \%$.

\section{CONCLUSIONS}


This research has applied IR thermography for the emissivity measurement and temperature measurement of the wall by making some improvements and corrections, provids some guidelines for the building measurement in situ. A solution of data processing is proposed that during a transient regime heat transfer period, the front face pulse response can be obtained by the temperature of the front face and heat flux of the same face of the wall via the TSVD method. The thermal properties of the wall can be characterized through the opotimization method based on the thermal quadrupoles model.

\section{REFERENCES}

[1] “U.S. Energy Information Administration, International Energy Outlook 2013, www.eia.gov/ieo/." Jul-2013.

[2] B. Yesilata and P. Turgut, "A simple dynamic measurement technique for comparing thermal insulation performances of anisotropic building materials," Energy Build., vol. 39, no. 9, pp. 1027-1034, Sep. 2007.

[3] A. Kylili, P. A. Fokaides, P. Christou, and S. A. Kalogirou, "Infrared thermography (IRT) applications for building diagnostics: A review," Appl. Energy, vol. 134, pp. 531-549, Dec. 2014.

[4] R. Albatici, A. M. Tonelli, and M. Chiogna, "A comprehensive experimental approach for the validation of quantitative infrared thermography in the evaluation of building thermal transmittance," Appl. Energy, vol. 141, pp. 218-228, Mar. 2015.

[5] Denis Maillet, Stephane Andre, Jean-Christophe Batsale, Alain Degiovanni, and Christian Moyne, Thermal Quadrupoles: Solving the Heat Equation through Integral Transforms. John Wiley \& Sons Ltd, 2000. 